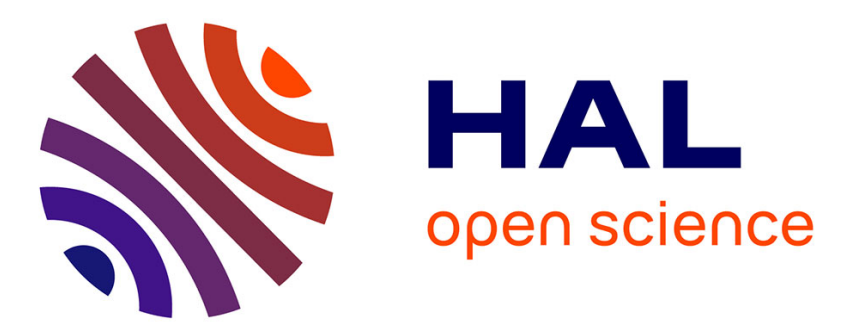

\title{
Generation of non-Gaussian tensor-valued random fields using an ISDE-based algorithm
}

Johann Guilleminot, Christian Soize

\section{To cite this version:}

Johann Guilleminot, Christian Soize. Generation of non-Gaussian tensor-valued random fields using an ISDE-based algorithm. ICOSSAR 2013, 11th International Conference on Structural Safety and Reliability, Columbia University, Jun 2013, New-York, United States. pp.1-6. hal-00806416

\section{HAL Id: hal-00806416 https://hal.science/hal-00806416}

Submitted on 3 Apr 2013

HAL is a multi-disciplinary open access archive for the deposit and dissemination of scientific research documents, whether they are published or not. The documents may come from teaching and research institutions in France or abroad, or from public or private research centers.
L'archive ouverte pluridisciplinaire HAL, est destinée au dépôt et à la diffusion de documents scientifiques de niveau recherche, publiés ou non, émanant des établissements d'enseignement et de recherche français ou étrangers, des laboratoires publics ou privés. 


\title{
Generation of non-Gaussian tensor-valued random fields using an ISDE-based algorithm
}

\author{
J. Guilleminot \& C. Soize \\ Laboratoire Modélisation et Simulation Multi Echelle, MSME UMR 8208 CNRS \\ Université Paris-Est, Marne la Vallée, France
}

\begin{abstract}
This work is concerned with the construction of a random generator for non-Gaussian tensorvalued random fields. Specifically, it focuses on the generation of the class of Prior Algebraic Stochastic Models associated with elliptic operators, for which the family of first-order marginal probability distributions is constructed using the MaxEnt principle. The strategy essentially relies on the definition of a family of diffusion processes, the invariant measures of which coincide with the target system of first-order marginal probability distributions. Those processes are classically defined as the unique stationary solutions of a family of Itô stochastic differential equations, the definition of which involves the construction of a family of normalized Wiener processes. The definition of the later allows spatial dependencies to be generated and the algorithm turns out to be very efficient for high probabilistic dimensions - it does not suffer from the curse of dimensionnality that is inherently exhibited by Gaussian chaos expansions, for instance. The algorithm is finally exemplified through the generation of a matrix-valued non-Gaussian random field.
\end{abstract}

\section{INTRODUCTION}

This work is concerned with the construction of a random generator for non-Gaussian tensor-valued random fields. More precisely, we consider the class of Prior Algebraic Stochastic Models (PASM) associated with elliptic operators, for which the family of first-order marginal probability distributions is constructed through the MaxEnt principle (Jaynes 1957). The construction of such a class has been pioneered - for the tensor case - in (Soize 2006), where the spatial dependencies are introduced through a nonlinear memoryless mapping acting on a set of $\mathbb{R}$ valued Gaussian homogeneous random fields (see (Guilleminot, Noshadravan, Soize, \& Ghanem 2011) and (Guilleminot \& Soize 2011) as well).

In this paper, we follow a similar path and extend the construction performed in (Soize 2006) to a more general case where the definition of the aforementioned mapping cannot be readily obtained in a closed form (Guilleminot \& Soize 2013). From a computational standpoint, the approach essentially relies on the definition of a family of diffusion processes, the invariant measures of which coincide by construction with the system of first-order marginal probability distributions to be prescribed. Those processes are defined as the unique stationary solutions of a family of Itô stochastic differential equations (ISDE). In particular, this family of ISDE involves the definition of a family of normalized Wiener processes in such a way that some spatial dependencies are generated through the generation procedure. We exemplify the efficiency of the approach through the generation of a vector-valued random field involved in multiscale analysis of linear microstructures.

\section{PROBLEM STATEMENT}

\subsection{Definition of a set of Gaussian stochastic germs}

Here, we denote by $\left\{\left\{\xi_{i}(\boldsymbol{x}), \boldsymbol{x} \in \mathbb{R}^{d}\right\}\right\}_{i=1}^{n}$ a set of independent second-order centered homogeneous realvalued Gaussian random fields, defined on some probability space $(\Theta, \mathcal{T}, P)$ and indexed by $\mathbb{R}^{d}$, such that for all $\boldsymbol{x}$ in $\mathbb{R}^{d}, \mathbb{E}\left\{\xi_{i}(\boldsymbol{x})^{2}\right\}=1, \forall i \in\{1, \ldots, n\}$ (with $\mathbb{E}$ the mathematical expectation). We further denote by $\left\{\boldsymbol{x} \mapsto \rho_{\xi_{i}}(\boldsymbol{x})\right\}_{i=1}^{i=n}$ the set of associated normalized continuous autocorrelation functions, with $\rho_{\xi_{i}}(\mathbf{0})=1$ for all $i$ in $\{1, \ldots, n\}$. Let $\left\{\boldsymbol{\xi}(\boldsymbol{x}), \boldsymbol{x} \in \mathbb{R}^{d}\right\}$ be the $\mathbb{R}^{n}$-valued Gaussian random field such that $\boldsymbol{\xi}(\boldsymbol{x}):=$ $\left(\xi_{1}(\boldsymbol{x}), \ldots, \xi_{n}(\boldsymbol{x})\right)$. Note that random field $\{\boldsymbol{\xi}(\boldsymbol{x}), \boldsymbol{x} \in$ $\left.\mathbb{R}^{d}\right\}$ is then mean-square continuous on $\mathbb{R}^{d}$.

\subsection{Definition of the random field}

Let $\{\boldsymbol{A}(\boldsymbol{x}), \boldsymbol{x} \in \Omega\}$ be the non-Gaussian $\mathbb{R}^{n}$-valued random field (with $\Omega$ an open bounded domain in $\mathbb{R}^{d}$ ) whose samples must be generated. At this stage, it is 
assumed that only the system of first-order marginal probability density functions (m.p.d.f.) of this field is given. Specifically, we assume that each probability density function (p.d.f.) of the aforementionned family reads

$\forall \boldsymbol{a} \in \mathbb{R}^{n}, \quad p_{\boldsymbol{x}}(\boldsymbol{a})=c_{\boldsymbol{x}} \exp \left(-\Phi_{\boldsymbol{x}}(\boldsymbol{a})\right)$

for all $\boldsymbol{x}$ in $\Omega$, with $c_{\boldsymbol{x}}$ the normalization constant. In Eq. (1), $\Phi_{x}: \mathbb{R}^{n} \rightarrow \mathbb{R}$ is a continuous potential function that is assumed to satisfy the following hypotheses:

H1 the function $\boldsymbol{a} \mapsto\left\|\nabla_{\boldsymbol{a}} \Phi_{\boldsymbol{x}}(\boldsymbol{a})\right\|_{\mathbb{R}^{n}}$ is a locally bounded function on $\mathbb{R}^{n}\left(\|\cdot\|_{\mathbb{R}^{n}}\right.$ being the Euclidean norm in $\mathbb{R}^{n}$ );

$\mathrm{H} 2 \lim _{R \rightarrow+\infty} \inf _{\|\boldsymbol{a}\|_{\mathbb{R}^{n}>R}} \Phi_{\boldsymbol{x}}(\boldsymbol{a})=+\infty ;$

H3 $\inf _{\boldsymbol{a} \in \mathbb{R}^{n}} \Phi_{\boldsymbol{x}}(\boldsymbol{a})=\Phi_{\boldsymbol{x}}^{\text {min }} \in \mathbb{R}$;

$\mathrm{H} 4 \int_{\mathbb{R}^{n}}\left\|\nabla_{\boldsymbol{a}} \Phi_{\boldsymbol{x}}(\boldsymbol{a})\right\|_{\mathbb{R}^{n}} p_{\boldsymbol{x}}(\boldsymbol{a}) \mathrm{d} \boldsymbol{a}<+\infty$.

Justifications of these assumptions can be found in the next section, where we define random field $\{\boldsymbol{A}(\boldsymbol{x}), \boldsymbol{x} \in \Omega\}$ through a nonlinear transformation (with memory) of the Gaussian germs.

\section{RANDOM GENERATOR}

\subsection{Definition of a family of normalized Wiener processes}

In this section, we introduce a family of normalized Wiener processes, indexed by $\Omega$, that allows for spatial depencies to be introduced. Let $\boldsymbol{W}=\left\{\boldsymbol{W}_{\boldsymbol{x}}(r)=\right.$ $\left.\left(W_{\boldsymbol{x}}^{(1)}(r), \ldots, W_{\boldsymbol{x}}^{(n)}(r)\right), \boldsymbol{x} \in \Omega, r \in \mathbb{R}^{+}\right\}$be a $\mathbb{R}^{n_{-}}$ valued centered second-order Gaussian random field such that

(i) for all $\boldsymbol{x}$ in $\Omega, \boldsymbol{W}_{\boldsymbol{x}}(0)=\mathbf{0}$ a.s.;

(ii) the generalized time-derivative of $\boldsymbol{W}$ is the cylindrical normalized Gaussian white noise $\boldsymbol{N}$ (see e.g. (Kree \& Soize 1986)).

The covariance generalized function $\left[\mathrm{C}_{B}\right]$ of $\boldsymbol{B}$ is then defined as

$$
\left[\mathbf{C}_{\boldsymbol{B}}\left(\boldsymbol{x}, \boldsymbol{x}^{\prime}, t+\tau, t\right)\right]_{i j}=\delta_{0}(\tau) \delta_{i j} \rho_{\xi^{i}}\left(\boldsymbol{x}-\boldsymbol{x}^{\prime}\right),
$$

for all $1 \leqslant i, j \leqslant n,\left(\boldsymbol{x}, \boldsymbol{x}^{\prime}\right)$ in $\Omega \times \Omega$ and $\tau \in \mathbb{R}$, with $\delta_{0}$ the Dirac generalized function at the origin of $\mathbb{R}$ and $\delta_{i j}$ the Kronecker delta. It follows that for all $\boldsymbol{x}$ fixed in $\Omega, \boldsymbol{W}_{\boldsymbol{x}}=\left\{\boldsymbol{W}_{\boldsymbol{x}}(r), r \geq 0\right\}$ is a normalized $\mathbb{R}^{n}$-valued Wiener process.

\subsection{ISDE-based generation algorithm}

Throughout this section, $\boldsymbol{x}$ is fixed in $\Omega$. Let $\left\{\left(\boldsymbol{U}_{\boldsymbol{x}}(r), \boldsymbol{V}_{\boldsymbol{x}}(r)\right), r \in \mathbb{R}^{+}\right\}$be a Markov stochastic process, defined on probability space $(\Theta, \mathcal{T}, \mathcal{P})$, with values in $\mathbb{R}^{n} \times \mathbb{R}^{n}$ and satisfying the following Itô stochastic differential equation (ISDE):

$$
\mathrm{d} \boldsymbol{U}_{\boldsymbol{x}}(r)=\boldsymbol{V}_{\boldsymbol{x}}(r) \mathrm{d} r,
$$

$$
\begin{aligned}
\mathrm{d} \boldsymbol{V}_{\boldsymbol{x}}(r)= & \left(-\nabla_{\boldsymbol{u}} \Phi_{\boldsymbol{x}}\left(\boldsymbol{U}_{\boldsymbol{x}}(r)\right)-\frac{1}{2} f_{\boldsymbol{x}} \boldsymbol{V}_{\boldsymbol{x}}(r)\right) \mathrm{d} r \\
& +\sqrt{f_{\boldsymbol{x}}} \mathrm{d} \boldsymbol{W}_{\boldsymbol{x}}(r),
\end{aligned}
$$

for all $r$ in $\mathbb{R}^{+}$, with $f_{x}$ a free parameter. In practice, the value of $f_{x}$ is selected in order to shorthen the transient regime. The above ISDE is completed with initial condition $\left(\boldsymbol{U}_{\boldsymbol{x}}(0), \boldsymbol{V}_{\boldsymbol{x}}(0)\right)=\left(\boldsymbol{U}_{\boldsymbol{x}}^{0}, \boldsymbol{V}_{\boldsymbol{x}}^{0}\right)$, where the probability distribution of random variable $\left(\boldsymbol{U}_{\boldsymbol{x}}^{0}, \boldsymbol{V}_{\boldsymbol{x}}^{0}\right)$ is assumed to be given.

When the potential function $\Phi_{\boldsymbol{x}}$ satisfies the hypotheses H1-H4, it can be shown (Soize 1994) (Soize 2008) that Eqs. (3-4) admit a unique stationary solution, which is a diffusion process with drift vector $\boldsymbol{b}(\boldsymbol{u}, \boldsymbol{v}) \in \mathbb{R}^{2 n}$ and diffusion matrix $[\sigma] \in \mathbb{M}_{2 n}^{+0}(\mathbb{R})$ $\left(\mathbb{M}_{2 n}^{+0}(\mathbb{R})\right.$ being the set of all the $2 n \times 2 n$ symmetric positive real matrices) such that

$\boldsymbol{b}(\boldsymbol{u}, \boldsymbol{v})=\left(\begin{array}{c}\boldsymbol{v} \\ -\nabla_{\boldsymbol{u}} \Phi_{\boldsymbol{x}}(\boldsymbol{u})-\frac{1}{2} f_{\boldsymbol{x}} \boldsymbol{v}\end{array}\right)$

and

$[\sigma]=\left(\begin{array}{cc}{\left[0_{n}\right]} & {\left[0_{n}\right]} \\ {\left[0_{n}\right]} & f_{\boldsymbol{x}}\left[I_{n}\right]\end{array}\right)$,

with $\left[0_{n}\right]$ and $\left[I_{n}\right]$ the $(n \times n)$ null and identity matrices, respectively. In addition, it can be shown that

$$
\lim _{r \rightarrow+\infty} \boldsymbol{U}_{\boldsymbol{x}}(r)=\boldsymbol{A}(\boldsymbol{x})
$$

in probability distribution (recall that $\boldsymbol{x}$ is fixed). Consequently, solving the ISDE allows sampling from the target marginal p.d.f. $p_{x}$ defined by Eq. (1), whereas the definition of the family of Wiener processes introduced in section 3.1 yields the spatial dependencies required for the random field modeling. It is worth stressing that proceeding this way, the correlation structure induced by the transformation (with memory) of the Gaussian germs cannot be constrained in a forward manner (in other words, the nonlinear mapping acting of the Gaussian fields is not defined imposing a match between the obtained correlation function and a target one). However, it does depend on the correlation functions retained for these germs, which can then be selected through an inverse analysis in order to impose a target correlation structure - see the application below. 


\subsection{Discretization scheme}

In this work, the ISDE is discretized using a StörmerVerlet (SV) algorithm (see (Hairer, Lubich, \& Wanner 2002) for a description of geometric integrators; see (Burrage, Lenane, \& Lythe 2007); see (Kloeden $\&$ Platen 1992) for an extensive survey of discretization schemes for SDE's).

For $k=1, \ldots, M-1$, we let $\boldsymbol{U}_{\boldsymbol{x}}^{k}=\boldsymbol{U}_{\boldsymbol{x}}\left(r_{k}\right), \boldsymbol{V}_{\boldsymbol{x}}^{k}=$ $\boldsymbol{V}_{\boldsymbol{x}}\left(r_{k}\right)$ and $r_{k}=(k-1) \Delta r$, where $\Delta r$ denotes the sampling step. For $k=1, \ldots, M-1$, the scheme writes

$$
\begin{aligned}
& \boldsymbol{U}_{\boldsymbol{x}}^{k+1 / 2}=\boldsymbol{U}_{\boldsymbol{x}}^{k}+\frac{\Delta r}{2} \boldsymbol{V}_{\boldsymbol{x}}^{k}, \\
& \boldsymbol{V}_{\boldsymbol{x}}^{k+1}=a_{1} \boldsymbol{V}_{\boldsymbol{x}}^{k}+a_{2} \boldsymbol{L}_{\boldsymbol{x}}^{k+1 / 2}+\sqrt{f_{\boldsymbol{x}}^{0}} \Delta \boldsymbol{W}_{\boldsymbol{x}}^{k+1}, \\
& \boldsymbol{U}_{\boldsymbol{x}}^{k+1}=\boldsymbol{U}_{\boldsymbol{x}}^{k+1 / 2}+\frac{\Delta r}{2} \boldsymbol{V}_{\boldsymbol{x}}^{k+1}, \\
& \boldsymbol{U}_{\boldsymbol{x}}^{1}=\boldsymbol{u}_{\boldsymbol{x}}^{0}, \quad \boldsymbol{V}_{\boldsymbol{x}}^{1}=\boldsymbol{v}_{\boldsymbol{x}}^{0},
\end{aligned}
$$

where

- the constants $a_{1}$ and $a_{2}$ are respectively defined as

$$
a_{1}=\frac{1-a}{1+a}, \quad a_{2}=\frac{\Delta r}{1+a},
$$

with $a=f_{x} \Delta r / 4$.

- $\Delta \boldsymbol{W}_{\boldsymbol{x}}^{k+1}=\boldsymbol{W}_{\boldsymbol{x}}\left(r_{k+1}\right)-\boldsymbol{W}_{\boldsymbol{x}}\left(r_{k}\right)$ denotes the increment of the Wiener process between $r_{k+1}$ and $r_{k}$, that is, $\Delta \boldsymbol{W}_{\boldsymbol{x}}^{k+1}$ is a second-order Gaussian centered $\mathbb{R}^{n}$-valued random variable with covariance matrix $\Delta r\left[I_{n}\right]$. More specifically, and following section 3.1 , we let

$$
\Delta \boldsymbol{W}_{\boldsymbol{x}}^{k+1}:=\sqrt{\Delta r} \boldsymbol{\xi}_{\boldsymbol{x}}\left(\theta_{k+1}\right),
$$

where $\boldsymbol{\xi}_{\boldsymbol{x}}\left(\theta_{k+1}\right)$ denotes the $(k+1)$-th independent realization of random variable $\boldsymbol{\xi}_{\boldsymbol{x}}:=\boldsymbol{\xi}(\boldsymbol{x})$.

- the $\mathbb{R}^{n}$-valued random variable $\boldsymbol{L}_{\boldsymbol{x}}^{k}$ is defined as

$$
\left(\boldsymbol{L}_{\boldsymbol{x}}^{k}\right)_{j}=-\left\{\frac{\partial \Phi\left(\boldsymbol{u} ; \boldsymbol{\lambda}_{\boldsymbol{x}}\right)}{\partial u_{j}}\right\}_{\boldsymbol{u}=\boldsymbol{U}_{\boldsymbol{x}}^{k}}
$$

for $j=1, \ldots, n$.

- $\boldsymbol{u}_{\boldsymbol{x}}^{0}$ and $\boldsymbol{v}_{\boldsymbol{x}}^{0}$ are arbitrary deterministic vectors.

Note that in the present case, the SV scheme turns out to be explicit and thus, conditionally stable. The convergence property given by Eq. (7) then reads as follows:

$\lim _{\Delta r \downarrow 0}\left(\lim _{k \rightarrow+\infty} \boldsymbol{U}_{\boldsymbol{x}}^{k}\right)=\boldsymbol{A}(\boldsymbol{x})$.

\subsection{Qualitative comparison with other sampling techniques}

From section 3.3, it is clear that the mapping could alternatively be defined using a Metropolis-like algorithm depending on a Gaussian transition kernel. However, the use of such an algorithm would rise two important issues that are worth pointing out. First of all, the definition of an optimal parametrization for the kernel would not be an easy task, even for moderate probabilistic dimensions. Note that the proposed algorithm does not present such a drawback: it is only parametrized by two quantities (regardless of the probabilistic dimension $n$ ), namely the integration step $\Delta r$ and the parameter $f_{\boldsymbol{x}}$, the values of which can be selected in a quite straightforward manner. In addition, the definition of the acceptance rates at all points is strongly related to the spatial resolution of the grid over which the random field has to be sampled. Clearly, the higher the number of points, the higher the acceptance rates. Therefore, generating the random field at many points would penalize the sampling quality, so that pursuing such an approach does not seem realistic for high probabilistic and spatial dimensions.

Similarly, and since the nonlinear transformation only aims at prescribing the system of first-order marginal p.d.f., a "pointwise" Gaussian chaos expansion (Wiener 1938) could be used as well. From a numerical standpoint, such a strategy would certainly be the most efficient in terms of computation time - as well as for subsequent analysis of uncertainty propagation. Nevertheless, and focusing on modeling aspects, this efficiency would be strongly penalized by the well-known curse of dimensionality. Moreover, the identification procedures for the chaos coefficients that have been proposed so far rely on imposing a match for the first- or second-order marginal distributions, hence making the preservation of the statistical dependences rather difficult as $n$ increases. Finally, one should note that numerical convergence problems can be encountered in practice, depending on the target p.d.f. that one wants to sample from.

Taking this into account, it is believed that the proposed algorithm does offer a good compromise, in the sense that it allows for an exact sampling with respect to the family of first-order marginal distributions - regardless of the probabilistic dimension -, at the expense of sampling stationary real-valued Gaussian random fields as many times as required to reach the stationary regime at all sampling points. Although this additional computational cost can appear as a limitation of the algorithm when a large number of iterations is necessary for reaching the invariant measures (especially when the Gaussian random fields are sampled at many points), we note that:

(i) the number of iterations can be reduced by choosing a larger integration step (which may degrade the quality of the approximations for the 
diffusions though);

(ii) this limitation is only related to computational ressources and can thus be reduced by adopting relevant numerical strategies (e.g. using parallel computing).

\section{APPLICATION}

In this section, the above algorithm is illustrated through the generation of a matrix-valued random field $\{[\boldsymbol{S}(\boldsymbol{x})], \boldsymbol{x} \in \Omega\}$ which is first defined in section 4.1. Numerical results are then presented in section 4.2.

\subsection{Prior algebraic stochastic model}

\subsubsection{Definition of the random field}

Let $\{[\boldsymbol{S}(\boldsymbol{x})], \boldsymbol{x} \in \Omega\}$ be a $\mathbb{M}_{6}^{+}(\mathbb{R})$-valued random field defined as follows. For all $\boldsymbol{x}$ in $\Omega$, the random matrix $[\boldsymbol{S}(\boldsymbol{x})]$ is assumed to be invariant under the action of a subgroup $\mathbb{O}$ of the special orthogonal group $\mathrm{SO}(3, \mathbb{R})$. From a mechanical point of view, the random field $\{[\boldsymbol{S}(\boldsymbol{x})], \boldsymbol{x} \in \Omega\}$ can be seen as an elasticity - or compliance - tensor random field exhibiting some material symmetry properties. In addition, it is assumed that the above random field satisfies the following properties:

$\mathbb{E}\{[\boldsymbol{S}(\boldsymbol{x})]\}=\left[I_{6}\right]$,

$\mathbb{E}\{\log (\operatorname{det}([\boldsymbol{S}(\boldsymbol{x})]))\}=\nu_{\boldsymbol{S}}(\boldsymbol{x}),\left|\nu_{\boldsymbol{S}}(\boldsymbol{x})\right|<+\infty$,

for all $\boldsymbol{x}$ in $\Omega$. From now on and without loss of generality, we assume for simplicity that $\mathbb{O}=\operatorname{SO}(3, \mathbb{R})$ (that is, $[\boldsymbol{S}(\boldsymbol{x})]$ is "isotropic" a.s.) and that parameter field $\boldsymbol{x} \mapsto \nu_{\boldsymbol{S}}(\boldsymbol{x})$ is constant over domain $\Omega$. We let $\nu_{\boldsymbol{S}}(\boldsymbol{x}):=\nu$ for all $\boldsymbol{x}$ in $\Omega$, with $\nu=-0.2$. For any $\boldsymbol{x}$ in $\Omega$, the random matrix $[\boldsymbol{S}(\boldsymbol{x})]$ is decomposed as

$[\boldsymbol{S}(\boldsymbol{x})]=3 S_{1}(\boldsymbol{x})[J]+2 S_{2}(\boldsymbol{x})[K]$,

where $[J]$ and $[K]$ constitute the classical matrix basis of the set of isotropic elasticity matrices (expressed using Voigt notation) (Walpole 1984). By construction, $\left\{S_{1}(\boldsymbol{x}), \boldsymbol{x} \in \Omega\right\}$ and $\left\{S_{2}(\boldsymbol{x}), \boldsymbol{x} \in \Omega\right\}$ are $\mathbb{R}_{*}^{+-}$ valued random fields (since $3 S_{1}(\boldsymbol{x})$ and $2 S_{2}(\boldsymbol{x})$ are the stochastic eigenvalues, with algebraic multiplicities 1 and 5 respectively, of $\mathbb{M}_{6}^{+}(\mathbb{R})$-valued random matrix $[\boldsymbol{S}(\boldsymbol{x})]$ ), hence making the above procedure irrelevant - the associated potential function does not satisfy the fundamental hypotheses $\mathrm{H} 1-\mathrm{H} 4$.

In order to circumvent this issue, we introduce an auxiliary random field $\{[\boldsymbol{A}(\boldsymbol{x})], \boldsymbol{x} \in \Omega\}$ such that

$[\boldsymbol{S}(\boldsymbol{x})]=\operatorname{expm}([\boldsymbol{A}(\boldsymbol{x})]), \quad \forall \boldsymbol{x} \in \Omega$,

where expm denotes the matrix exponential. It can be shown that $[\boldsymbol{A}(\boldsymbol{x})]$ admits a similar decomposition, namely

$[\boldsymbol{A}(\boldsymbol{x})]=A_{1}(\boldsymbol{x})[J]+A_{2}(\boldsymbol{x})[K]$,

where $\left\{A_{1}(\boldsymbol{x}), \boldsymbol{x} \in \Omega\right\}$ and $\left\{A_{2}(\boldsymbol{x}), \boldsymbol{x} \in \Omega\right\}$ are now $\mathbb{R}$-valued random fields.

\subsubsection{First-order marginal p.d.f.}

Upon substituting Eq. (19) in Eqs. (16-17), it can be shown that the target MaxEnt marginal p.d.f. $p_{\boldsymbol{A}(\boldsymbol{x})}$ of random variable $\boldsymbol{A}(\boldsymbol{x})=\left(A_{1}(\boldsymbol{x}), A_{2}(\boldsymbol{x})\right)$ takes the form (Guilleminot \& Soize 2013)

$p_{\boldsymbol{A}(\boldsymbol{x})}(\boldsymbol{a})=p_{A_{1}(\boldsymbol{x})}\left(a_{1}\right) \times p_{A_{2}(\boldsymbol{x})}\left(a_{2}\right)$

for all $\left(a_{1}, a_{2}\right) \in \mathbb{R} \times \mathbb{R}$, where

$p_{A_{1}(\boldsymbol{x})}\left(a_{1}\right)=c_{1} \exp \left(-\lambda_{1} \exp \left\{a_{1}\right\}-\lambda a_{1}\right)$

and

$p_{A_{2}(\boldsymbol{x})}\left(a_{2}\right)=c_{2} \exp \left(-5\left(\lambda_{2} \exp \left\{a_{2}\right\}+\lambda a_{2}\right)\right)$,

$c_{1}$ and $c_{2}$ being the two normalization constants. The parameters $\lambda_{1}>0, \lambda_{2}>0$ and $\lambda<0$ are such that the random variable $[\boldsymbol{S}(\boldsymbol{x})]$ defined by Eq. (19) and Eqs. (20) to (23) satisfies the constraints given by Eqs. (16-17). Finally, it is seen that for the case under consideration, the two-dimensional ISDE given by Eqs. (3-4) reduces to two uncoupled one-dimensional ISDE's, each of which involving a potential function defined with respect to either Eq. (22) or Eq. (23).

Remark. In practice, the random field $\{[\boldsymbol{S}(\boldsymbol{x})], \boldsymbol{x} \in$ $\Omega\}$ thus constructed can be used as a prior stochastic model with almost sure symmetry properties for the modeling of three-dimensional elasticity tensor random fields - see (Guilleminot \& Soize 2013) for details.

\subsection{Numerical results}

For illustration purposes, a one-dimensional application is considered, with $\Omega=] 0,100[$ (in $\mathrm{mm}$ ). Furthermore, we set $f_{\boldsymbol{x}}=9.5$ for all $x \in \Omega$ and $\Delta r=0.001$.

\subsubsection{Definition of the input correlation functions}

Here, we consider Gaussian stochastic germs defined either by a squared-sinc or by an exponential correlation function, namely

$\rho_{\xi_{i}}^{s q s}(\tau)=\left(\frac{2 L_{i}}{\pi \tau}\right)^{2} \sin ^{2}\left(\frac{\pi \tau}{2 L_{i}}\right)$

or

$\rho_{\xi_{i}}^{\exp }(\tau)=\exp \left(-|\tau| / L_{i}\right)$

for all $\tau$ in $\mathbb{R}$ and $i \in\{1, \ldots, n\}$, with $L_{i}>0$ (here, $n=2$ ). In both cases, parameter $L_{i}$ corresponds to the spatial correlation length of the random germ, that is:

$\int_{0}^{+\infty}\left|\rho_{\xi_{i}}(\tau)\right| \mathrm{d} \tau=L_{i}$.

The plot of these correlation functions is shown in Fig. 1, for $L_{i}=20 \mathrm{~mm}$. We now let $L_{1}=L_{2}=20$ mm. 


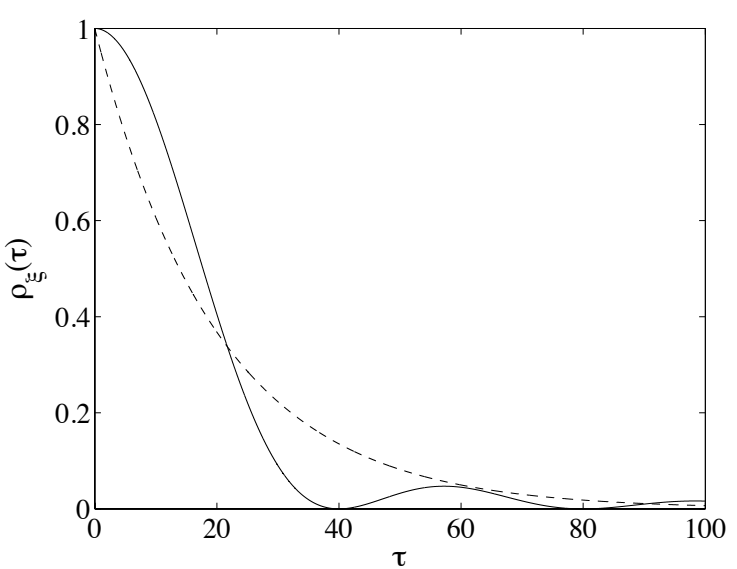

Figure 1: Plot of $\tau \mapsto \rho_{\xi^{i}}^{s q s}(\tau)$ (solid line) and $\tau \mapsto \rho_{\xi^{i}}^{e x p}(\tau)$ (dashed line) for $L_{i}=20 \mathrm{~mm}$.

\subsubsection{Convergence results}

Since the target p.d.f. defined by Eq. (21) does not depend on $x$, the convergence towards the stationary measure can be investigated once at some arbitrary point in $\Omega$. Below, this convergence is characterized through the convergence of the ergodic estimator for the second-order moment of random variable $\left\|\boldsymbol{U}_{\boldsymbol{x}}\right\|_{\mathbb{R}^{2}}$ :

$\operatorname{ConvMes}\left(N_{\text {iter }}\right):=\frac{1}{N_{\text {iter }}} \sum_{k=1}^{N_{\text {iter }}}\left\|\boldsymbol{U}_{\boldsymbol{x}}^{k}\right\|_{\mathbb{R}^{2}}^{2}$.

The plot of ConvMes is shown in Fig. 2. It is seen that

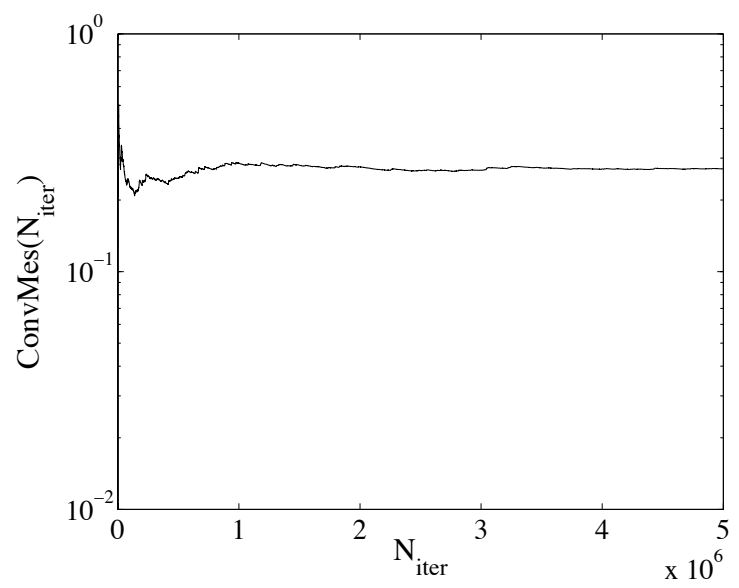

Figure 2: Convergence towards the stationary solution: plot of $N_{\text {iter }} \mapsto \operatorname{ConvMes}\left(N_{\text {iter }}\right)$ in semilog scale.

a very good convergence to the stationary solution is obtained for $N_{\text {iter }} \geqslant 2 \times 10^{6}$.

\subsubsection{Statistical results}

The plot of $p_{A_{1}(x)}$ and $p_{A_{2}(x)}$ at some arbitrary point is shown in Fig. 3, hence illustrating the nongaussianity of random variables $A_{1}(x)$ and $A_{2}(x)$. The plot of correlation functions for random fields $\left\{A_{1}(x), x \in\right] 0,100[\}$ and $\left\{A_{2}(x), x \in\right] 0,100[\}$ are shown in Figs. 4 and 5 for input correlation functions of squared-sinc and exponential types respectively.

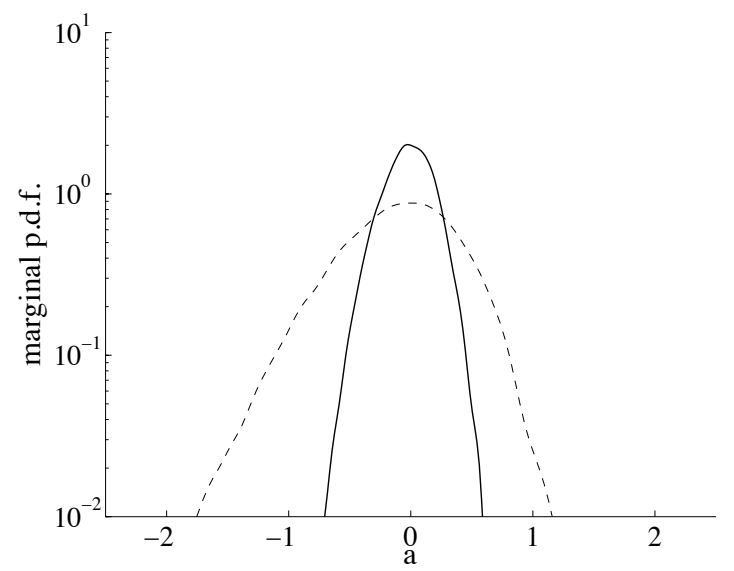

Figure 3: Plot of p.d.f. $a \mapsto p_{A_{1}(x)}(a)$ (dashed line) and $a \mapsto$ $p_{A_{2}(x)}(a)$ (solid line) in semilog scale.

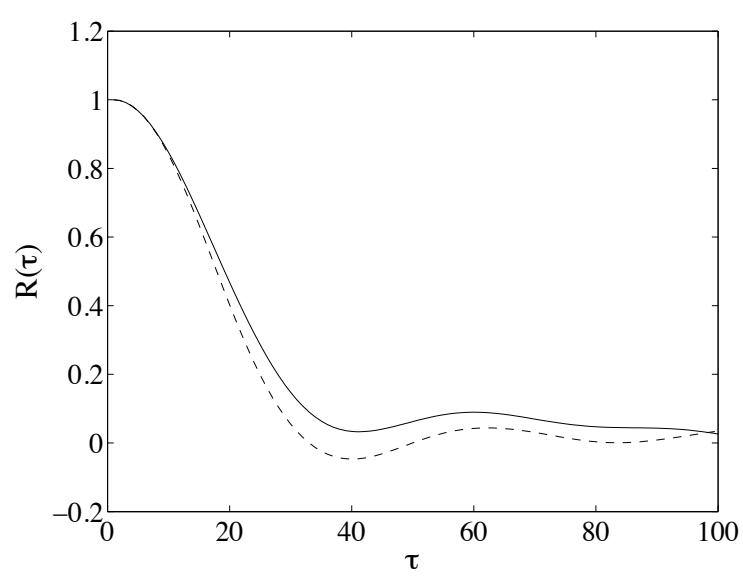

Figure 4: Plot of correlation function $\tau \mapsto R(\tau)$ associated with random fields $\left\{A_{1}(x), x \in\right] 0,100[\}$ (dashed line) and $\left\{A_{2}(x), x \in\right] 0,100[\}$ (solid line) for a squared-sinc input correlation function.

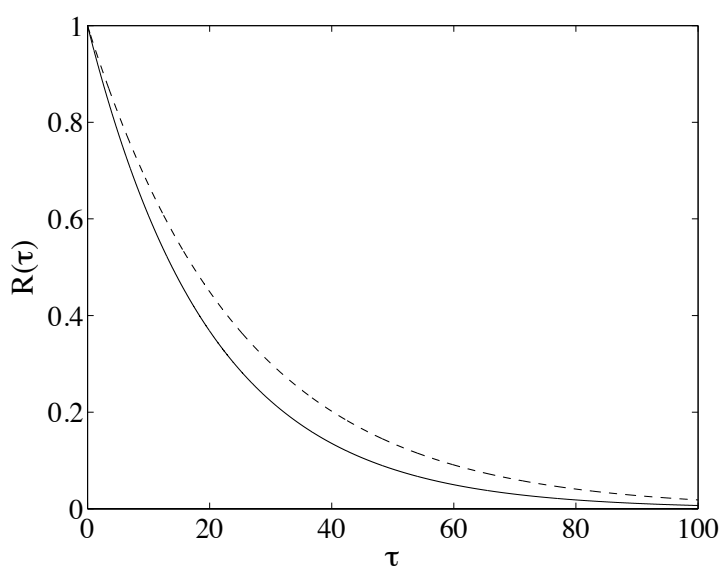

Figure 5: Plot of correlation function $\tau \mapsto R(\tau)$ associated with random fields $\left\{A_{1}(x), x \in\right] 0,100[\}$ (dashed line) and $\left\{A_{2}(x), x \in\right] 0,100[\}$ (solid line) for an exponential input correlation function.

In both cases, it is seen that the output correlation functions present a shape that is similar to that of the input correlation functions. This fact is of particular interest whenever one is interested in imposing a target shape for the output correlation functions. Finally, it is observed that the spatial correlation lengths are slightly modified (with a typical magnitude of about 
$10 \%)$.

\section{CONCLUSIONS}

We have presented the construction of a random generator for a class of non-Gaussian tensor-valued random fields, for which the family of first-order marginal probability distributions is defined through the MaxEnt principle. The strategy essentially relies on the definition of a family of diffusion processes, the invariant measures of which coincide with the target system of first-order marginal probability distributions. Those processes are specifically defined as the unique stationary solutions of a family of Itô stochastic differential equations. A particular definition of the associated family of driving normalized Wiener processes finally allows spatial dependencies to be generated. The parametrization of the algorithm turns out to be independent of both the probabilistic and spatial dimensions and makes the numerical strategy especially suitable for sampling in high probabilistic dimensions. It should be noticed that the proposed algorithm can readily be used for sampling any nonGaussian vector-valued random field, provided that the associated family of first-order marginal p.d.f. takes the form given by Eq. (1) and that the associated family of potential functions satisfy the hypotheses $\mathrm{H} 1-\mathrm{H} 4$. Finally, the algorithm is exemplified through the generation of a matrix-valued non-Gaussian random field.

\section{ACKNOWLEDGEMENTS}

The financial support of the French National Research Agency (Agence Nationale de la Recherche) is gratefully acknowledged. The work of J. Guilleminot is supported by ANR grants ANR-2010-BLAN-0904 and ANR-12-JS09-0001-01. The work of C. Soize is supported by ANR grant ANR-2010-BLAN-0904.

\section{REFERENCES}

Burrage, K., I. Lenane, \& G. Lythe (2007). Numerical methods for second-order stochastic differential equations. SIAM J. Sci. Comput. 29(1), 245-264.

Guilleminot, J., A. Noshadravan, C. Soize, \& R. G. Ghanem (2011). A probabilistic model for bounded elasticity tensor random fields with application to polycrystalline microstructures. Computer Methods in Applied Mechanics and Engineering 200, 1637-1648.

Guilleminot, J. \& C. Soize (2011). Non-Gaussian positivedefinite matrix-valued random fields with constrained eigenvalues: Application to random elasticity tensors with uncertain material symmetries. International Journal of Numerical Methods in Engineering 88, 1128-1151. DOI:10.1002/nme.3212.

Guilleminot, J. \& C. Soize (2013). Stochastic model and generator for random fields with symmetry properties: Application to the mesoscopic modeling of elastic random media. Multiscale Modeling \& Simulation. submitted.
Hairer, E., C. Lubich, \& G. Wanner (2002). Geometric Numerical Integration. Structure-Preserving Algorithms for Ordinary Differential Equations. Berlin: Springer.

Jaynes, E. T. (1957). Information theory and statistical mechanics. Physical Review 106/108(4/2), 620-630/171-190.

Kloeden, P. E. \& E. Platen (1992). Numerical Solution of Stochastic Differentials Equations. Berlin: Springer.

Kree, P. \& C. Soize (1986). Mathematics of Random Phenomena. Dordrecht, Holland: Reidel Publishing Company.

Soize, C. (1994). The Fokker-Planck Equation for Stochastic Dynamical Systems and its Explicit Steady State Solutions. Singapore: World Scientific.

Soize, C. (2006). Non-gaussian positive-definite matrix-valued random fields for elliptic stochastic partial differential operators. Computer Methods in Applied Mechanics and Engineering 195, 26-64.

Soize, C. (2008). Construction of probability distributions in high dimension using the maximum entropy principle: Applications to stochastic processes, random fields and random matrices. International Journal of Numerical Methods in Engineering 76, 1583-1611.

Walpole, L. J. (1984). Fourth-rank tensors of the thirty-two crystal classes: Multiplication tables. Proc. R. Soc. Lond. A 391, 149-179.

Wiener, N. (1938). The Homogeneous Chaos. American Journal of Mathematics 60(4), 897-936. 